\title{
D oenças crônicas não-transmissíveis no Brasil: repercussões do modelo de atenção à saúde sobre a seguridade social
}

\author{
Chronic non-communicable diseases in Brazil: \\ the health care system and the social security sector
}

Aloyzio Achutti 1

M aria Inês Reinert Azambuja 2

1 Universidade Federal do Rio Grande do Sul (UFRGS). Academia Sul-Rio-Grandense de Medicina.

Av. Bastian 212, 90130-020,

Porto Alegre RS.

achutti@cardiol.br 2

Departamento de M edicina

Social da Faculdade de

Medicina da UFRGS.

\begin{abstract}
Social security comprehends governmental and societal actions on the rights to the social insurance, to social service, and to health care itself. This article sketches each one of these elements. M any of this group disease have common risk factors, demanding continuous attention. Comparing our population with that from the US, it is possible to observe that they are quite identical until the 15 to 24 years old, while the American population is twofold bigger from 35 to 44 years old and more than four times above the 75. This difference explains why the number of deaths by CNCD is still very low in Brazil compared with US: our population is younger and dies before, by other causes. The ageing process of our population, particularly through reduction of the early mortality, will be followed by the increased proportion of the CNCD and their impact in the social security. Health care as well as social insurance and social service are suffering the pressure of the power hegemony, from economy and culture of the global market. Trying to fore cast a possible future scenario for the social security in Brazil, it is being discussed the urgent necessity to reform the country budget, aiming the financial balance.
\end{abstract}

Key words Chronic non-communicable diseases, Health care system, Demography, Social security
Resumo A seguridade social envolve ações do poder público e da sociedade sobre direitos à previdência social, à assistência social e à própria saúde. Este artigo traça um esboço de cada um desses elementos. M uitas doenças crônicas nãotransmissíveis têm fatores de risco comuns e demandam assistência continuada de serviços. Comparando-senossa população com a dos EUA, vê-se que é praticamente do mesmo tamanho até a faixa dos 15 aos 24 anos. A americana é duas vezes maior dos 35 aos 44 anos e mais de quatro vezes maior acima dos 75 anos. Tais diferenças explicam porque o número de mortes por DCNT é muito mais baixo no Brasil: nossa população é mais jovem emorre antes. $\mathrm{Na}$ medida em que 0 processo de envel hecimento avance, especialmente, via redução da mortalidade precoce, aumentará a prevalência das DCNT e sua repercussão na seguridade social. Assim como a atenção à saúde, a previdência social e a assi stência social sofrem pressões políticas, econômicas e culturais. $\mathrm{Na}$ tentativa de imaginar um cenário futuro possível para a seguridade social no Brasil discute-se a necessidade de reformular o orçamento do País, visando ao equilíbrio financeiro.

Palavras-chave Doenças crônicas não-transmissíveis, M odelo assistencial, D emografia, Seguridade social 


\section{Introdução}

Este tema contém vários desafios, a começar pelo exercício de previsões, caminho sempre difícil de seguir dentro dos padrões habituais da literatura epidemiológica. Serve, entretanto, para levantar questionamentos e suscitar discussões de utilidade imediata, contribuindo para ajustar preconceitos, tentar esclarecer confusões originadas em conflitos ideológicos, ajudando a enfrentar dificuldades naturais na tradução do discurso para a formulação de uma política de saúde, e sua ulterior aplicação prática.

0 tema é, por natureza, transetorial, o que não surpreende em se tratando de saúde e seus problemas, cuja causalidade em geral se situa fora do setor em que se verifica o impacto. Esta característica torna mais difícil não somente sua análise e compreensão, mas principalmente sua utilização no planejamento de possíveis intervenções e adoção de políticas adequadas que satisfaçam todos os atores envolvidos.

Como seguridade social envolve ações do poder público e da sociedade sobre os direitos à previdência social, à assistência social e à própria saúde, no âmbito deste artigo cabe apenas um esboço sobre a contribuição de cada um destes elementos e suas interações na avaliação do impacto das doenças crônicas não-transmissíveis (DCNT) sobre todo o sistema - sem a pretensão de medir custo-benefício de intervenções sobre este grupo de doenças.

\section{As doenças crônicas não-transmissíveis}

O grupo das DCNT compreende majoritariamente doenças cardiovasculares, diabetes, câncer e doenças respiratórias crônicas. Muitas doenças deste grupo têm fatores de risco comuns, e demandam por assistência continuada de serviços e ônus progressivo, na razão direta do envelhecimento dos indivíduos e da população.

Há mais de trinta anos, este conjunto de doenças é reconhecido como importante não somente em países industrializados (OMS, 1972). M as nos últimos três anos, com base em recortes de um estudo publicado por Murray e Lopez em 1996, seguiram-se vários artigos, publicados internacionalmente, chamando a atenção para um possível deslocamento da epidemia de doenças crônicas, das economias industrializadas em direção aos países menos desenvolvidos (Whelan, 2002; World Health Report, 2002). Com base nesta expectativa, tem-se preconizado mudanças nos sistemas nacionais de saúde, que no lugar de cuidarem predominantemente de condições agudas, passem a se organizar para a atenção continuada de doenças crônicas.

Em reunião realizada no Rio de Janeiro em novembro de 2003, com representantes do Ministério da Saúde, da Organização PanAmericana de Saúde, Organização M undial da Saúde e da Organização das Nações Unidas para a Agricultura e Alimentação, foi dito que este conjunto de doenças é responsável por $60 \%$ das mortes e incapacidade em todo 0 mundo, numa escala progressiva, podendo chegar a $73 \%$ de todas as mortes em 2020. Em 2001, no Brasil, as DCNT foram responsáveis por $62 \%$ de todas as mortes e $39 \%$ de todas as hospital izações registradas no Sistema Ú nico de Saúde (OPAS/O M S, 2004).

As premissas subjacentes a este "alerta de epidemia" nos documentos citados são:

1. as DCNT são decorrentes do estilo de vida ocidental;

2. a adoção deste estilo de vida por populações em vias de desenvolvimento determina uma modificação de seu perfil de morbidade, aumentando a importância das DCNT (transição epidemiológica);

3. estes fatores de risco são os mesmos ao longo do tempo e no espaço;

4. seus efeitos são modificáveis por intervenção sanitária;

5. a redução na mortalidade por DCNT verificada nos EUA e em outros países semel hantes deveu-se à intervenção com sucesso sobre estes fatores de riscos.

$\mathrm{Na}$ verdade, esta última premissa não é tão definitiva pois, conforme M athers (2002), existem três hipóteses para explicar a surpreendente queda da mortalidade entre os mais vel hos registrada nos EUA e em outros países desenvolvidos nos últimos 30 anos:

1. A hipótese da expansão da morbidade (Gruenberg, 1977; Kramer, 1980) postula que o declínio na mortal idade é devido à redução nas taxas de letalidade das doenças, e não à redução na incidência ou na sua progressão. N esse caso, a queda na letalidade seria basicamente por avanços e extensão de atendimento médico qualificado focado nas fases mais avançadas da evolução da doença. Tais avanços incluem a disseminação dos centros de trata- 
mento intensivo, as modernas tecnologias diagnósticas e de tratamento clínico ou a cirurgia das coronárias. Conseqüentemente, o declínio na mortalidade seria acompanhado por um aumento na prevalência de doenças crônicas e de incapacidade;

2. A hipótese da compressão da morbidade proposta por Fries (1989) postula que, com a expectativa de vida adulta se aproximando de seu limite biológico, se a inci dência de doenças incapacitantes puder ser retardada para idades posteriores, então a morbidade será comprimida para um período mais curto de vida. Esta é a concepção que justificaria a intervenção sanitária precoce e continuada preconizada pelas organizações internacionais (promoção da saúde, prevenção primária e secundária);

3. A hipótese proposta por Manton (1982, 1987) sugere que o declínio na mortalidade pode ser causado, ao menos parcialmente, por redução na letalidade, mas que teria havido de fato diminuição da incidência e do potencial da intensidade de progressão das doenças. A queda na incidência e uma evolução aparentemente mais benigna dos casos remanescentes - talvez associada à variação nos mecanismos etiopatogênicos predominantes ao longo do tempo (Azambuja, 2004) - teriam sido as responsáveis pelo progressivo aumento na proporção de saudáveis e longevos na população.

Com o envelhecimento de nossa população e a persistência na exposição aos fatores de risCo, aumentando a ocorrência de DCNT no Brasil, quais seriam as repercussões, sobre a seguridade social, considerando as alternativas das intervenções sanitárias sugeridas a partir das teorias que explicariam sua queda no Primeiro Mundo?

1. Se o objetivo for o declínio na mortalidade às custas de redução da letalidade apenas, ou seja, por um modelo de cuidado focado na atenção secundária e terciária, o setor saúde trocaria custos (teóricos) de acompanhamento e medicalização de longo prazo, por ampliação do acesso a medicamentos e tecnologias concentrados em um período menor de tempo. A repercussão na seguridade dependeria da sobrevida que um maior acesso a cuidados médicos com mais tecnologia e qualidade pudesse somar ao cuidado já dispensado hoje a este grupo de pacientes, uma vez que, apesar de suas falhas e baixa cobertura, este é o modelo de atenção dominante em nosso país.

2. Se o objetivo for a compressão da morbidade, teríamos certamente um aumento de custo para o Sistema de Saúde, que teria de investir em detecção precoce, controles freqüentes e, provavelmente, dispensação de medicamentos ( prevenção primária e secundária) ao longo de décadas, o que não eliminaria a necessidade de fármacos e tecnologia mais intensivamente aplicada na evolução dos casos, mesmo supondo que se reduza o número dos que dela necessitem. Este é o modelo que tem sido preconizado para enfrentarmos a ameaça de "epidemia" de DCNT. Na previdência, esperar-se-ia um deslocamento do impacto econômico, da concessão de benefícios precoces (por incapacidade temporária ou definitiva para o trabalho), para mais tempo de prestação continuada de aposentadoria, por incremento na longevidade.

3. Se, entretanto, a queda na mortalidade (verificada nos EUA e outros países com características semelhantes) fosse devida à queda na incidência destas doenças (M anton) - hipótese provável e de causa ainda em polêmica (Azambuja, 2004) - nada impediria que o mesmo fenômeno estivesse ocorrendo em todo o mundo, e que o deslocamento esperado da epidemia de fato não estivesse ocorrendo. N este caso, uma maior proporção da população seria saudável ao longo da vida, e teria vida mais longa. Os custos para o Sistema de Saúde cairiam, e os custos previdenciários aumentariam somente pelo aumento da longevidade.

O bviamente esta avaliação do impacto de modelos assistenciais no atendimento das DC NT sobre a seguridade social é simplista, por ser unidimensional. Pressupõe que não haja outros determinantes (competing-causes de morbi-mortalidade e outros determinantes demográficos e econômicos) para o desempenho do sistema.

\section{A transição demográfica}

O bserva-se em todo o mundo, o Brasil incluído, um envelhecimento populacional que certamente causa impacto na política e nos orçamentos da seguridade social, voltada particularmente para os estratos demográficos de mais idade e para os incapacitados. Se a ocorrência das DCNT aumenta com o envelhecimento da população, cada vez mais deverá aumentar 0 ônus delas decorrente entre os beneficiários do seguro social (Datasus, 2004; M PAS, 2002).

$\mathrm{Na}$ tentativa de delinear um cenário no qual compareçam os determinantes demográ- 
ficos e os de saúde, seria preciso contar com os seguintes elementos: a natalidade, a mortalidade infantil, a morbi-mortalidade do adulto jovem (trabalhador mais exposto às causas externas), do adulto de meia-idade, no qual começam a aparecer mais as DCNT, e do adulto idoso, que vai determinar seu período de sobrevida depois da aposentadoria ou da instalação de limitação ou incapacidade para prover o próprio sustento, bem como de seus dependentes.

O bviamente tais elementos não são independentes e, por sua vez, estão determinados por uma série de outros mais de ordem sociocultural eque não comparecem claramente nas categorias demográficas e nosológicas: a determinação da freqüência, precocidade e importância de muitas doenças do grupo DCNT estariam sendo definidas desde a gestação, como na hipótese de Barker (2003), fazendo com que os sobreviventes da mortalidade infantil estivessem sujeitos a mais este desafio; o risco de acidentes de trabalho e a capacidade de se defender deles dependem da natureza e da organização do mesmo e do treinamento e cultura do trabalhador; o risco da violência é maior nos grupos sociais menos privilegiados e em ambientes por eles freqüentados.
No Brasil, a mortalidade ainda ocorre precocemente, e especialmente nas regiões mais pobres (Figura 1). M as, embora a longevidade dos cidadãos ainda seja baixa, comparativamente com o Primeiro M undo, o conjunto da população brasileira já envelheceu, pois há proporcionalmente menos crianças hoje do que há 30 anos. Este envelhecimento, no entanto, é pequeno comparado com a situação de outras populações. Nos EUA, não só a sobrevida individual é maior como também repercutem na estrutura etária da população as ondas migratórias e a geração "baby-boom" nascida no póssegunda-guerra. A figura 2 mostra que, enquanto a população dos EUA é praticamente do mesmo tamanho que a do Brasil até a faixa dos 15 aos 24 anos, ela é duas vezes maior na faixa dos 35 aos 44, e mais de quatro vezes maior acima dos 75 anos. Esta diferença explica por que o número de mortes por DCNT ainda é muito mais baixo no Brasil do que nos EUA: a nossa população é mais jovem, e morre antes, por outras causas.

$\mathrm{Na}$ medida em que o processo de envelhecimento da nossa população avançar, especialmente via redução da mortalidade precoce, deverá aumentar a prevalência das DCN T esua repercussão na seguridade social.

\section{Figura 1}

Distribuição proporcional da mortalidade geral por grupos etários, nas Regiões Norte e Sul do Brasil e nos EUA, em 1999.

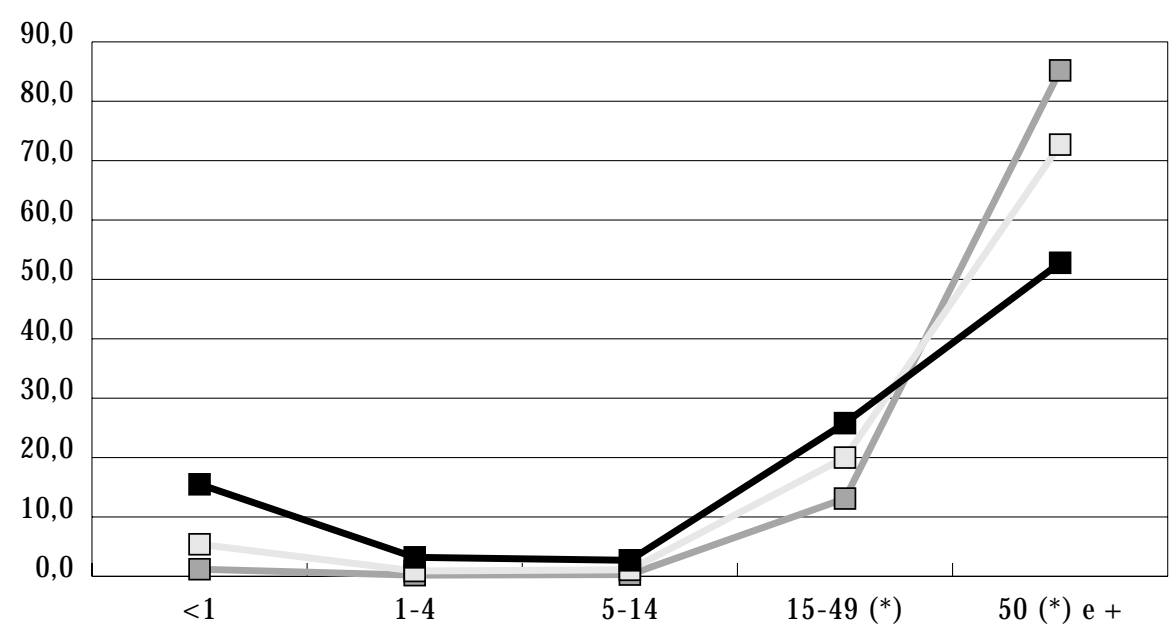

EUA

Brasil/Região Sul

Brasil/Região Norte

(*) EUA, 15-45 e $55+$ 
Figura 2

Tamanho relativo da população dos EUA com relação à do Brasil, em sucessivas faixas etárias.

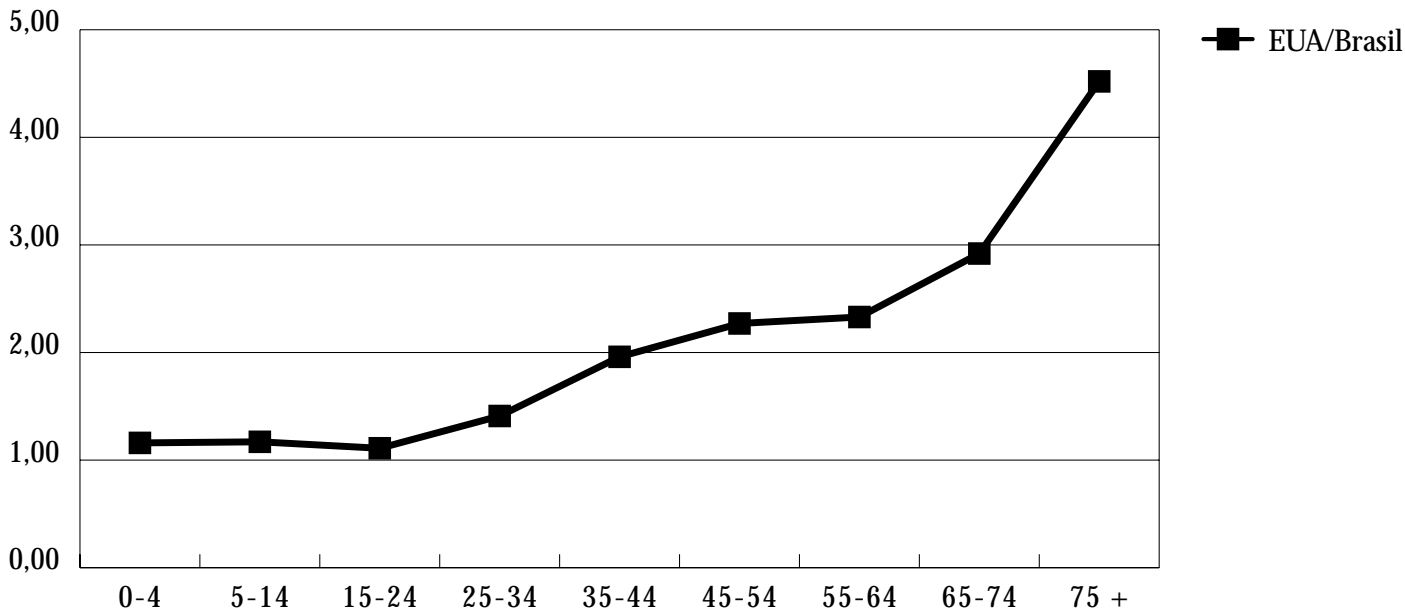

\section{A seguridade social}

A Seguridade Social compreende um conjunto integrado de ações de iniciativa dos poderes públicos e da sociedade, destinado a assegurar o direito relativo à saúde, à previdência e à assistência social (Previdência Social, 1999).

Assim como a atenção à saúde, a previdência e a assistência social em nosso meio e no resto do mundo sofrem as pressões da atual hegemonia do poder, da economia e da cultura, pelo mercado globalizado. Todas estão entremeadas por interesses econômicos, e não podem ser discutidas fora deste contexto.

A previdência está baseada no conceito de seguro, ou seja, em um contrato individual que afirma a existência de um direito pessoal do contribuinte (ou afiliado ao sistema) a um benefício futuro em razão e na proporção de sua contribuição presente. São elementos críticos no cálculo do valor desta contribuição: a estrutura etária da população, sua longevidade e a taxa de emprego. Já o conceito de seguridade baseia-se em um contrato social, pelo qual o direito aos benefícios aparece como um direito da cidadania. É esta a concepção que norteia a concessão de aposentadorias aos trabal hadores rurais e a concessão de benefícios previstos na LOAS - Lei Orgânica da Previdência Social - para idosos (mais de 65 anos) e portadores de deficiência comprovada em qualquer idade, com renda familiar per capita mensal inferior a $25 \%$ do salário mínimo. No custeio hoje do sistema, podem ser apontados como nós críticos o grau de desemprego e informalidade; o rebaixamento do salário dos trabalhadores mais jovens quando comparado aos dos mais velhos que estão em vias de se aposentar; o custeio das aposentadorias dos trabalhadores rurais; 0 grande número de aposentadorias urbanas por idade, para as quais bastam 15 anos de contribuição; e o número crescente de benefícios por incapacidade temporária ou definitiva para o trabalho.

Segundo o PNAD/IBGE, em 2001 existiam 40,7 milhões de trabalhadores ocupados que não contribuíam para a Previdência Social. Este contingente correspondia a $57,7 \%$ da população ocupada privada, isto é, quase $58 \mathrm{em}$ cada 100 trabal hadores ativos sem vínculo contributivo com a Previdência (2003). A tendência que se observa na economia brasileira tem sido no sentido do agravamento deste quadro.

Em março de 2004, o INSS emitiu aproximadamente 22 milhões de ben efícios (Boletim Estatístico da Previdência Social, 2004). Destes, as aposentadorias por tempo de contribuição foram $16 \%$, e as por idade (em boa parte para 
trabalhadores rurais) $55 \%$ do total. Já a soma das aposentadorias por invalidez, auxíliosdoença, e benefícios assistenciais para não contribuintes (LOAS e renda mensal vitalícia por invalidez) - na maior parte decorrentes de agravos à saúde ou suas seqüelas, somaram $23 \%$. 0 INSS ainda não disponibiliza estatísticas que permitam estabelecer as causas básicas dos benefícios concedidos por incapacidade temporária ou invalidez permanente. As informações disponíveis são esparsas.

Em Porto Alegre (Boff et al., 2002), a análise de um total de 6.898 benefícios E-31 (auxílio-doença comum por incapacidade temporária para o trabalho) concedidos em 1998 (não incluídos os acidentes do trabalho) mostrou que as "causas externas" responderam por 1.486 (22\%); a "convalescen ça após cirurgia", por 1.181 (17\%); e as "condições clínicas", por 4.119 (61\%) (Previdência Social, 2003). Comparadas a estudo realizado no Brasil em 1986 (M endes, 1995), as causas externas passaram da quarta para a primeira posição com determinante de incapacidade temporária para 0 trabalho. D os $61 \%$ de afastamentos temporários por incapacidade decorrente de doença clínica $(60 \%)$, as doenças do aparelho circulatório ocuparam o 3 ㅇ lugar (16,2\%), após doenças osteomusculares $(24,8 \%)$ e as doenças mentais $(18,9 \%)$. No grupo das doenças do aparelho circulatório, as causas de afastamento foram, pela ordem, doença hipertensiva, doença isquêmica do coração, doenças cerebrovasculares, e doenças das veias. É provável que as aposentadorias por invalidez tenham outra distribuição relativa de causas básicas, com maior proporção de DCNT entre os casos.

\section{0 modelo de atenção à saúde}

Se as transições demográfica e epidemiológica têm potencial de impacto sobre a seguridade social, a efetividade de nossa política sanitária e de nosso sistema assistencial precisaria ser também contemplada. Um modelo de atenção à saúde pressuporia a existência de algo bem definido, estruturado e testado. Sabe-se que por enquanto nossas ações de saúde se desenvolvem num ambiente nebuloso, imaturo e de difícil avaliação, dificultando a sua designação como um verdadeiro modelo.

Em que pese $o$ enorme esforço de nossos bem-intencionados planejadores de saúde, não se pode ainda falar em sistema de atenção à saúde com identidade própria, com integração dos diversos níveis administrativos e de jurisdição, com regulamentação do privado, do governamental e público, com clara atribuição de competências e responsabilidades, com implementação adequada de serviços e avaliação de resultados, com remuneração adequada e treinamento de pessoal, com uma política definida em cima de prioridades levantadas no nível local das populações a serem atendidas.

Mesmo contando com um bom planejamento para um modelo de atenção à saúde, nossa heterogeneidade (e doença social) 0 comprometeria, dificultando 0 acesso ao diagnóstico precoce e à possibilidade de uma assistência de qualidade no diagnóstico e na obtenção dos recursos terapêuticos.

Especialmente em se tratando de DCNT, o intervalo de tempo entre a intervenção e a medida de sua efetividade costuma ser mais longo, medido em décadas ou até gerações, dependendo do grau de exigência e do interesse em afastar possíveis fatores de confusão.

O planejamento de intervenções sobre os fatores de risco clássicos para DCNT, extrapolados de outros países, e orquestrados por interesses ao menos duvidosos, pode ser também questionado, e neste caso o interval o entre a intervenção e o seu impacto deve ser ainda maior. É preciso também considerar que os custos destas intervenções mais precoces na história natural, embora aparentemente mais baratos no nível individual, podem ser muito el evados, considerando-se a extensão da cobertura exigida e a necessidade de compreender múltiplos outros aspectos interdependentes. Entre estes, certamente está incluída a educação ou, indo mais além, o desenvolvimento humano integral.

Estas considerações não significam uma fuga da responsabilidade em responder ao tema proposto, mas de justificar o grau de incerteza que se oculta atrás de uma equação intersetorial, aparentemente bem formulada. Visa também provocar os especialistas para tecerem seus comentários e apresentarem suas hipóteses e respostas.

Para prever o impacto sobre a seguridade social, a tendência da transição demográfica já se mostra evidente, mas nosso modelo assistencial, para dizer de seu potencial impacto sobre a transição epidemiológica, ainda precisa ser construído. 


\section{Conclusões}

- A transição demográfica em países como o nosso tende a tornar mais crítica a desproporção entre contribuintes e beneficiários do sistema de previdência social. Com o aumento do desemprego e a redução da massa salarial dos contribuintes, menos trabalhadores com salários mais baixos terão que sustentar mais aposentados com salários mais altos por mais tempo. 0 que está ocorrendo hoje, perversamente, é que a aposentadoria dos mais velhos é com freqüência a única fonte estável de renda de toda a família (filhos e netos), e os velhos são usurpados do direito de utilizá-la a seu critério.

- A vulnerabilidade do sistema de seguro social nas condições aqui descritas traz maior responsabilidade aos políticos e gestores do sistema de seguro social e aumenta a culpabilidade daqueles que o fraudam.

- A promoção de mais empregos no mercado formal e a regularização das contribuições devidas ao Seguro Social estão entre as soluções de curto prazo para minimizar os desequilíbrios atuais, que tendem a se aprofundar em um cenário de transição demográfica e epide- miológica sobreposto à longa crise econômica que estamos vivendo.

- Como o SUS ainda tem deficiências graves, os custos da incapacidade recaem pesadamente sobre a previdência e a assistência social, já que os pacientes permanecem em benefício por muito tempo, aguardando exames, procedimentos ou acesso a um especialista para o tratamento adequado. Uma integração maior entre a Previdência e Assistência Social e o SU S é fundamental.

- Os programas de prevenção de DCNT e de promoção da saúde deveriam contar com estudos relacionados com suas conseqüências de mais longo prazo, para medir sua efetividade e orientar medidas reguladoras do impacto no sistema de seguridade social.

- A avaliação das repercussões de diferentes alternativas de intervenção nas DCNT sobre o sistema de seguridade social deve levar em conta a estrutura demográfica e o padrão de morbidade da nossa população, o estado de organização do SUS e de sua integração com a previdência e a assistência social, e as possibilidades de acesso e limitações de financiamento do sistema, na atual conjuntura econômico-social do País.

\section{Colaboradores}

A Achutti e M IR Azambuja participaram igualmente na concepção, discussão e redação final do texto.

\section{Referências bibliográficas}

Pedro Soares 2004. Com Lula, renda cai e desemprego cresce. Disponível em <http://www1.folha.uol.com.br/ fsp/dinheiro/fi2401200402.htm >. Acesso em 22/4/2004.

Azambuja M I 2004. Spanish flu and early 20th-century expansion of a coronary heart disease-prone subpopulation. Texas Heart Institute Journal 31:14-21.

Barker D 2003. The midwife, the coincidence, and the hypothesis. British M edical Journal 327:20-27.

Boff BM , Leite DF \& Azambuja MI. M orbidade subjacente à concessão de benefício por incapacidade temporária para o trabalho. Revista de Saúde Pública 36:337-342.

Boletim Estatístico da Previdência Social 9(3). Disponível em <http://www.previdenciasocial.gov.br/docs/10.xls>. Acesso em 22/4/2004.

Datasus 2004. Dados de morbidade hospitalar e mortalidade. Disponível em <http://www.datasus.gov.br> Acesso em 20/04/2004.
Epping-Jordan JÁ 2001. The challenge of chronic conditions: WHO responds. [Editorial]. British Medical Journal 323:947-948.

FriesJ 1989. Aging, natural death and the compression of morbidity. New England Journal of M edicine 303:130135.

Gruenberg EM 1977. The failures of success. Milbank Memorial Fund Quarterly - Health and Society 55:324.

IBGE 2004. Síntese de Indicadores Sociais 2003. Comunicação Social, 13/04/2004. Rio de Janeiro.

Kramer M 1980. The rising pandemic of mental disorders and associated diseases and disabilities. Acta Psychiatric Scandinavica 62:282-297.

Lenfant C 2001. Can we prevent cardiovascular diseases in low- and middle-income countries? Bulletin of WHO 79:980-982.

Manton KG 1982. Changing concepts of morbidity and 
mortality in the elderly population. M ilbank M emorial Fund Quarterly - Health and Society 60:183-244.

$M$ anton KG 1987. The linkage of health status changes and disability. Comprehensive Gerontology 1:16-24.

$M$ athers CD 2002. Health expectancies: an overview and critical appraisal, pp. 177-204. In CJL M urray et al. (Eds). Summary measures of population health. WHO, Genebra

M endes R 1995. Aspectos conceituais das patologias do trabalho, pp. 33-47. In R M endes (org.). Patologia do trabalho. Atheneu. São Paulo.

Nissinen A, Berrios X \& Puska P 2001. Community-based non-communicable disease interventions: lessons from developed countries for developing ones. Bulletin of WHO 79:963-970.

OM S 1972. Conclusões da Conferência M undial da Saúde 1972. Genebra.

OPAS/OM S 2004. Disponível em <http://portalweb02. saude.gov.br/saude/visualizar_texto.cfm?idtxt=17098> Acesso em 14/03/2004.

Previdência Social 2003. Cobertura da Previdência Social no Brasil: perfil dos não-contribuintes e evolução re cente. Informe da Previdência Social, março 2003.
Disponível em <http://www.previdenciasocial.gov.br/ docs/inf_marco03.pdf >. Acesso em 22/4/2004.

Reddy KS 2001. Neglecting cardiovascular disease is unaffordable. Bulletin of WHO 79:985.

Regulamento da Previdência Social: Decreto no 3.048, de 6 de maio de 1999. Disponível em http://www.dji. com.br/decretos/1999003048/regulamento da previ dencia social.htm> Acesso em 6/06/2004.

Santana RLF, Pouchain GC \& Bissi LF 2002. A Previdência Social e o Censo 2000: perfil dos idosos. Informe dePrevidência Social, setembro de 2002. 14(9).

Secretaria de Previdência Social (MPAS) 2002. Saldo previdenciário e arrecadação. Informe de Previdência Social, setembro de 2002 (9), Brasília.

Whelan J 2002. WHO calls for countries to shift from acute to chronic care. N ews. British M edical Journal 324:1237.

Winker M A 2002. Aging in the 21st century: a call for papers. JAM A 287(10).

World Health Report 2002. Chapter one. Protecting the people. Disponível em <http://www.who.int/whr/ 2002/chapter1/en/>. Acesso em 8/6/2004. 